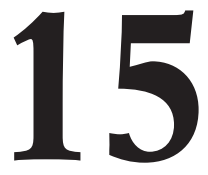

\title{
EFICACIA DEL ENTRENAMIENTO ESPACIAL EN PRIMARIA Y SECUNDARIA: TODOS APRENDEN
}

\author{
(EFFECTIVENESS OF SPATIAL TRAINING IN ELEMENTARY AND \\ SECONDARY SCHOOL: EVERYONE LEARNS)
}

Antonio Rodán

Universidad San Pablo CEU

Pedro R. Montoro

UNED

Agustín Martínez-Molina

Universidad Autónoma de Madrid

María José Contreras

UNED

DOI: $10.5944 / e d u c X X 1.30100$

\section{Cómo referenciar este artículo/How to reference this article:}

Rodán, A., Montoro, P.R., Martínez-Molina, A. y Contreras, M.J. (2022). Eficacia del entrenamiento espacial en primaria y secundaria: todos aprenden. Educación XX1, 25(1), 381-406. https://doi.org/10.5944/educXX1.30100

Rodán, A., Montoro, P.R., Martínez-Molina, A., \& Contreras, M.J. (2022). Effectiveness of spatial training in elementary and secondary school: everyone learns. Educación XX1, 25(1), 381-406. https://doi.org/10.5944/educXX1.30100

\section{RESUMEN}

El procesamiento visoespacial es clave para lograr, entre otros, un rendimiento óptimo en actividades académicas. En el ámbito de la cognición espacial se ha encontrado que la práctica con tareas espaciales puede reducir la brecha de género en este tipo de razonamiento. Sin embargo, no siempre un aumento de las puntuaciones espaciales llega a compensar las diferencias que existen entre participantes con mayores y menores habilidades espaciales. De acuerdo con estudios previos sobre diferencias individuales y maleabilidad en cognición espacial, se necesitan estudios comparables en niños, niñas y adolescentes utilizando el mismo método de evaluación y entrenamiento. En este trabajo se analiza, en 39 estudiantes de Educación Primaria (estudio 1: 17 niños y 22 niñas) y en 21 de Educación 
Secundaria (estudio 2: 11 chicos y 10 chicas), el perfil de evolución a través de las diferentes sesiones de un entrenamiento en rotación mental (RM), así como el grado de mejora producido en función de su capacidad espacial de partida, analizando el factor género. En ambos grupos, se aplicó un entrenamiento espacial (Programa de Entrenamiento en Rotación Mental) durante tres sesiones consecutivas con una duración promedio de 35 minutos por sesión. Para ambos grupos de edad, los participantes con bajo nivel espacial se beneficiaron en una proporción similar que aquellos participantes con más recursos espaciales. Este resultado se replicó para ambos sexos. Esta investigación servirá como punto de partida para promover e implementar entrenamientos adaptativos y personalizados, y así poder ayudar a los que menos capacidades espaciales tienen. Este tipo de intervenciones ganarían en eficacia y podrían maximizar el potencial educativo en los grupos más desfavorecidos.

\section{PALABRAS CLAVE}

Rotación mental, entrenamiento, diferencias de género, ganancias de ejecución, estrategias educativas

\section{ABSTRACT}

Visuospatial processing is key to achieve optimal performance in academic activities, among others. In the field of spatial cognition, it has been found that practice with spatial tasks can reduce the gender gap in this type of reasoning. However, an increase in spatial scores does not always compensate the differences that exist between participants with greater and lesser spatial abilities. According to previous studies on individual differences and malleability in spatial cognition, comparable studies are needed in children and adolescents using the same evaluation and training method. 39 students of Primary Education (study 1: 17 boys and 22 girls) and 21 of Secondary Education (study 2: 11 boys and 10 girls) participated in this study. The evolution profile through three sessions in a mental rotation (RM) training was analyzed, as well as the degree of improvement produced based on their initial spatial performance, analyzing the gender factor. In both groups, a spatial intervention (Mental Rotation Training Program) was applied during three consecutive sessions with an average duration of 35 minutes per session. For both age groups, participants with a low spatial level benefited at a similar rate to those participants with more spatial resources. This result was replicated for both sexes. This research will serve as a starting point to promote and implement adaptive and personalized training, and thus be able to help those who have fewer spatial skills. These 
types of interventions would become more effective and could maximize educational potential in the most disadvantaged groups.

\section{KEYWORDS}

Mental rotation, training, gender differences, achievement gains, educational strategies

\section{INTRODUCCIÓN}

Los contenidos espaciales suelen estar presentes en áreas académicas relacionadas con la ciencia, tecnología, ingenierías o matemáticas, de manera que las habilidades viso-espaciales predicen en alguna medida el éxito en estas disciplinas (Humphreys, Lubinski y Yao, 1993). Algunos meta-análisis han mostrado que el entrenamiento visoespacial puede mejorar el rendimiento de esta habilidad (ej., Uttal et al., 2013). Investigadores vinculados al área de la educación han incorporado intervenciones espaciales en el aula para que estas habilidades se "impregnen" en disciplinas académicas como las matemáticas (ej., Hawes et al., 2017; Lowrie et al., 2017).

Algunas intervenciones para mejorar funciones cognitivas han analizado las ganancias producidas tras un entrenamiento en función de las capacidades iniciales, encontrando mayores beneficios en aquellos individuos con niveles más bajos en memoria de trabajo (Karbach et al., 2015) y rendimiento lector en escolares (García-Madruga et al., 2013), o en atención dividida en adultos (Baniqued et al., 2014).

Las intervenciones centradas en cognición espacial, más concretamente en Rotación Mental (RM), han mostrado diferencias en el rendimiento a favor del sexo masculino (ej., Linn y Petersen, 1985). Sin embargo, la práctica con tareas espaciales puede minimizar la brecha de género igualando esta habilidad en ambos sexos tras un entrenamiento en escolares (De Lisi y Wolford, 2002; Ehrlich et al., 2006), adolescentes (Neubauer et al., 2010) y universitarios (Cherney et al., 2014).

Fernández-Méndez et al. (2020) analizaron las ganancias diferenciales según el nivel espacial de partida en preescolares de 3 y 5 años. Solo en el grupo de 5 años los participantes con peor habilidad inicial incrementaron más su habilidad de RM tras un entrenamiento. En la adolescencia también se han realizado algunos estudios sobre los efectos de un entrenamiento según diferencias individuales de partida, encontrando resultados dispares. David (2012) encontró, en estudiantes de 14 años que practicaron con 
videojuegos relacionados con la RM, la Visualización (Vz) y las relaciones espaciales, que los estudiantes con puntuaciones bajas mejoraban significativamente más su rendimiento en RM respecto a aquellos con mejores habilidades iniciales después de la práctica con videojuegos. Sin embargo, la autora no encontró un efecto de género, ni una interacción del nivel inicial por género. Los hallazgos de Bergner y Neubauer (2011) en estudiantes de 15 años con altas capacidades en aptitud visoespacial, sugirieron que el grupo de chicas con un menor rendimiento espacial mejoraba más en la precisión de la tarea tras el entrenamiento, aunque no en los Tiempos de Respuesta (TR). En el contexto de los déficits cognitivos, Wiedenbauer y Jansen-Osmann (2007), encontraron que los niños y niñas con edades comprendidas entre los 8 y los 14 años diagnosticados de espina bífida se beneficiaban considerablemente del entrenamiento, mejorando sus TR y su proporción de aciertos en la tarea de RM informatizada hasta igualar el rendimiento al de los niños sanos.

Las diferencias individuales en RM también se han analizado en personas adultas, con un entrenamiento con el videojuego Tetris, encontrando resultados dispares. Mientras que unos autores han visto que jugadores inexpertos se benefician tanto como los expertos en este videojuego (Sims y Mayer, 2002), otros han encontrado que los que tienen peores habilidades iniciales obtienen mayores incrementos hasta equipararse en las últimas sesiones con quienes tenían altas habilidades espaciales (Terlecki et al., 2008). Otra investigación de Contreras et al. (2018), también encontraron que estudiantes universitarios con menores habilidades iniciales en $\mathrm{Vz}$ mejoraban significativamente más respecto a sus compañeros con mejores capacidades iniciales.

En resumen, aunque la mayoría de los estudios apuntan hacia una mejoría más pronunciada tras un entrenamiento en aquellos participantes que inicialmente exhiben una menor habilidad espacial inicial, algunas investigaciones encuentran que participantes con mejor o peor nivel se benefician por igual de un entrenamiento espacial. Por otra parte, un metaanálisis influyente, que ha confirmado la maleabilidad de las habilidades espaciales, hace un llamamiento sobre la falta de investigaciones en diversas edades con un método de entrenamiento similar para poder hacer comparables los resultados (véase Uttal et al., 2013, para una revisión). Dada la escasa literatura sobre trabajos que analicen el rendimiento individual con tareas de RM, nos planteamos buscar más evidencias utilizando por primera vez un método análogo en evaluación y entrenamiento, en dos etapas educativas que son cruciales, Primaria (infancia) y Secundaria (adolescencia). Fomentar el razonamiento espacial en Educación Primaria podría ser clave para que los niños y niñas alcancen un nivel óptimo de razonamiento visoespacial en etapas posteriores, como la adolescencia, 
en la que los estudiantes deben determinar qué camino académico desean seguir, siendo esto crucial para su futura carrera profesional. Por tanto, intervenciones educativas de carácter espacial que tengan lugar antes o durante la adolescencia podrían ser relevantes, especialmente cuando la presencia de mujeres es escasa en carreras STEM (Science, Technology, Engineering and Mathematics), donde el razonamiento espacial parece jugar un importante papel.

\section{Objetivos e hipótesis}

El objetivo de los dos estudios (estudiantes de Primaria y de Secundaria) del presente trabajo fue analizar el progreso a lo largo de tres sesiones de entrenamiento en una tarea de RM, así como el rendimiento en función de una alta o baja capacidad espacial medida en la prueba criterio aplicada, controlando que los dos grupos fueran equiparables en inteligencia general. Se esperaba que, tras el entrenamiento, los participantes con una habilidad inicial más baja en la prueba espacial alcanzasen un nivel similar a los que partían de un nivel más alto. La ejecución se valoró mediante las medidas de proporción de aciertos y tiempo de respuesta (TR) en cada una de las sesiones de la tarea. A continuación, se muestran los dos estudios realizados con 39 estudiantes de primaria y 21 de secundaria, pertenecientes únicamente a los grupos que realizaron el entrenamiento en RM (GE, en adelante). Los resultados sobre las diferencias pretest y postest, así como las mejoras producidas en los grupos experimental y control en la habilidad espacial tras el entrenamiento, pueden verse en Rodán et al. (2019) para el grupo de primaria, y en Rodán et al. (2016) para el grupo de secundaria.

\section{ESTUDIO 1}

\section{Método}

\section{Participantes}

En este estudio participaron 39 estudiantes $(M=7.68$ años, $D T=$ 0.66; 17 niños y 22 niñas) de segundo curso de Educación Primaria de dos escuelas públicas ("CEIP Escuelas Aguirre" y "CEIP Leopoldo Alas"), ubicadas en una zona céntrica de Madrid, con un nivel sociocultural medioalto. Estos fueron estudiantes que pertenecían al GE de un estudio previo donde se comparó el rendimiento entre un grupo entrenado con otro grupo control —GC, en adelante— no entrenado (Rodán et al., 2019). 


\section{Materiales}

Matrices progresivas de Raven (versión SPM; Raven, Court, y Raven, 1996)

Evalúa la inteligencia general desde los 6 años. El test consta de 60 ejercicios divididos en 5 series con 12 elementos cada uno, y se ejecuta sin límite de tiempo, aunque la duración media de la prueba es de 40-90 minutos. Cada lámina tiene 6 u 8 opciones de respuesta, con solo una opción correcta. La máxima puntuación posible es de 60 puntos. La fiabilidad dos mitades es .90, mientras que el test-retest oscila entre .83 y .90 (Seisdedos, 1995).

\section{Prueba de aptitud espacial "E" del EFAI-1}

Es una subprueba de la batería de Evaluación Factorial de Aptitudes Intelectuales (Santamaría et al., 2005), validada para 7 a 10 años. Evalúa la capacidad para imaginar mentalmente movimientos y transformaciones de un objeto en el espacio, siendo considerada como una aproximación al factor de procesamiento visual, "Gv" —Lohman, 1996- (p. 17). Tiene 30 ejercicios con cuatro opciones de respuesta cada uno, donde se decide cuál de los cuatro estímulos respuesta al rotarlos encaja a modo de puzle en un target de referencia. La duración de la prueba es de seis minutos, y puntuación máxima es de 30 puntos. La fiabilidad de este test es de.86 (ver un ejemplo de ítem en Rodán et al., 2019).

\section{Programa de Entrenamiento de Rotación Mental (PERM)}

El PERM está formado por 480 ensayos, y cada uno consta de un molde blanco sobre una caja gris (target de referencia) situado en el lado izquierdo de la pantalla, y de dos figuras bidimensionales localizadas a la derecha, enumeradas como "1" y "2", con una forma idéntica al molde. La tarea del PERM consiste en imaginar transformaciones y rotaciones, decidiendo cuál de los dos estímulos "1" o "2" coincide con el molde si se gira o se rota. Solo uno de los dos estímulos encaja dentro del molde (Figura 1). La dificultad intra e inter sesión de la tarea fue manipulada según las características de los estímulos - figuras cercanas y concretas o figuras abstractas-, y el número de ensayos de mayor dificultad se incrementaba con el avance de las sesiones. Durante la realización del PERM, el participante debía responder a cada uno de los ensayos, de forma que el programa registraba 450 ensayos de los 480 que se presentaban (los 10 primeros ensayos de cada sesión eran de familiarización con la tarea). En cada sesión se computaron los aciertos y el tiempo de respuesta de cada ensayo. Se utilizó el software informático E-Prime versión 1.2 (Psychology Software Tools, 2002) para la programación, presentación de estímulos y recopilación de datos del PERM 
(para información adicional sobre el PERM, véase el artículo de Rodán et al., 2019).

\section{Figura 1}

Ejemplo de un ensayo utilizado en el PERM del estudio 1
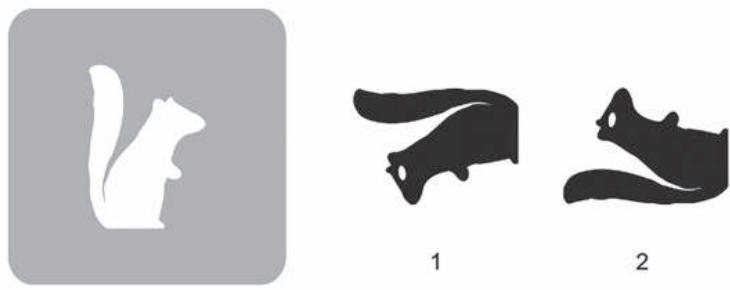

\section{Procedimiento}

Las pruebas se administraron en días consecutivos, con una sesión por día, durante un periodo total de cinco días (un día para el pretest, tres días de entrenamiento y un día para el postest).

El primer día (fase pretest) se administraron colectivamente primero las Matrices progresivas de Raven y después el EFAI-1, con un descanso entre ambas de cinco minutos. Se realizó en el aula habitual de los/as alumnos/ as con cuatro examinadores, y las mesas se organizaron individualmente.

Los siguientes tres días, cada participante realizó la tarea del entrenamiento (PERM) de manera individual en un ordenador durante tres sesiones consecutivas. Después de los 10 ensayos de pre-entrenamiento se llevaron a cabo los 150 ensayos de la tarea propiamente dicha, dividida en dos bloques de 75 presentaciones, con un descanso de 5 minutos entre ambos bloques. Los experimentadores destacaron la importancia de la precisión durante todo el entrenamiento, insistiendo en que podían tomarse todo el tiempo necesario para asegurar una respuesta correcta. Todos los ensayos debían responderse de manera forzada puesto que no se contemplaba la posibilidad de respuestas en blanco. El número de ensayos por sesión presentados en el PERM fue de 5850 (150 ensayos x 39 participantes). Tras eliminar algunos de ellos por anticipación o exceso de tiempo consumido, se analizaron un total de 5723 (97.8\%), 5656 (96.7\%) y $5655(96.7 \%)$ en las sesiones S1, S2 y S3, respectivamente. Descontando estos ensayos eliminados, el tiempo mínimo de realización fue de $311.4 \mathrm{seg}$. (5.19 min.), 335.4 seg. (5.59 min.) y $223.2 \mathrm{seg}$. (3.72 min.), mientras que el tiempo máximo fue de $1176 \mathrm{seg}$. (19.6 min.), $1416 \mathrm{seg}$. (23.6 min.) y 1290 seg. (21.5 min.) para la S1, S2 y S3, respectivamente. 
Finalmente, en el último día (fase postest), se administraron las pruebas Matrices Progresivas de Raven y EFAI-1 en las mismas condiciones que en la fase pretest.

Los tutores legales de los niños firmaron un consentimiento informado, según la Declaración de Helsinki, cumpliendo la normativa de protección de datos y la aprobación del Comité de Ética de la UNED.

Análisis de datos

Para confirmar que los grupos fueran comparables en la inteligencia según el nivel de habilidad espacial (alta: “^HE”; baja: “ЏHE”), y en ambos sexos, se realizó un ANOVA de un factor para la medida obtenida en el Raven en la fase pretest. Para examinar el perfil de evolución a través de las diferentes sesiones de entrenamiento en función del nivel de HE y del sexo, se llevó a cabo un ANOVA mixto de medidas repetidas $2 \times 2 \times 2$ con "nivel HE" (bajo, alto) y "sexo" (niños, niñas) como factores inter-sujetos, y con medidas repetidas "sesión" (sesión 1, sesión 2 y sesión 3) como factor intrasujetos. Este análisis se aplicó a las variables dependientes "proporción de aciertos" ( $\mathrm{n}^{\circ}$ aciertos/total ensayos en cada sesión) y "tiempo de respuesta" (tiempo entre la presentación del ensayo y la respuesta correcta), en la fase de entrenamiento.

Finalmente, para analizar las ganancias obtenidas en la capacidad espacial (EFAI-E) en función del nivel de HE y del sexo, se llevó a cabo un ANOVA de medidas repetidas $2 \times 2 \times 2$ con "nivel HE" (bajo, alto) y "sexo" (niños, niñas), como factores inter-sujetos, y con "medidas repetidas prepost” (pretest, postest) como factor intra-sujetos.

Los análisis estadísticos se realizaron con el programa estadístico informático SPSS, versión 24.0 (IBM Corp., 2016) con un nivel de significación de .05 .

\section{RESULTADOS}

\section{Análisis preliminares}

El análisis preliminar mostró que ambos grupos ( $\downarrow \mathrm{HE}$ y $\uparrow \mathrm{HE})$ no diferían en sus puntuaciones en la prueba de inteligencia de la fase pretest. Tampoco se encontraron diferencias de sexo para todo el grupo, ni la interacción entre los factores grupo y sexo. 


\section{Rendimiento durante el PERM y ganancias obtenidas en la habilidad de RM}

El nivel de HE ( $\downarrow$ HE y $\uparrow$ HE) de los participantes se determinó según las habilidades observadas en la prueba espacial (EFAI-E) en la fase pretest, utilizando la mediana como punto de corte, que en este estudio fue de 9 puntos.

En el análisis del rendimiento del PERM, se excluyeron todos los ensayos con TR inferiores a 500 ms. (Wiedenbauer y Jansen-Osmann, 2007), y los TR superiores a 60 segundos de todos los participantes, de forma que finalmente se eliminaron 516 ensayos (2.94\% del total). El promedio de TR (en segundos) para cada participante se calculó solo para las respuestas correctas siguiendo el mismo procedimiento utilizado en estudios clásicos de referencia para el análisis de la ejecución en tareas de Rotación Mental (Shepard y Metzler, 1971; Voyer y Jansen, 2016).

La Tabla 1 muestra los análisis descriptivos de la proporción de aciertos y de los TR de los ensayos acertados. El ANOVA mixto 2 (sexo) x 2 (nivel HE) para la proporción de aciertos, reveló un efecto principal del nivel HE en la sesión $1\left[F(1,35)=5.67, M S E=.073, p=.023, \eta^{2}{ }_{p}=.14\right]$, en la sesión $3\left[F(1,35)=4.251, M S E=.068, p=.047, \eta^{2}=.11\right]$, y para el total de todas las sesiones $\left[F(1,35)=4.19, M S E=.050, p=.048, \eta^{2}=.11\right]$, de forma que los participantes con $\uparrow \mathrm{HE}$ obtuvieron una mayor proporción de aciertos en estas tres condiciones: [AC-S1: $\downarrow$ HE: $M=.78$, $\uparrow \mathrm{HE}: M=.87$, diferencia de medias $=.09, p=.02]$, [AC-S3: $\downarrow$ HE: $M=.72$, $\uparrow$ HE: $M=.80$, diferencia de medias $=.08, p=.04$ ], [AC-TOTAL: $\downarrow$ HE: $M=.75$, $\uparrow$ HE: $M=.82$, diferencia de medias $=.07, p=.04]$. No se encontraron otros efectos principales, ni interacciones entre factores. El ANOVA de medidas repetidas 3 (sesión) x 2 (sexo) x 2 (nivel HE), solo mostró un efecto significativo en el factor sesión $\left[F(2,70)=18.9, M S E=.056, p<.001, \eta^{2}=.35\right]$. La proporción de aciertos disminuyó progresivamente de la $\mathrm{S} 1$ a la $\mathrm{p} 3$, y las comparaciones por pares mostraron una mayor proporción de aciertos en la S1 respecto a la S2 y S3 $[\mathrm{S} 1: M=0.83 ; \mathrm{S} 2: M=.76 ; \mathrm{S} 3: M=.76$; diferencia de medias $\mathrm{S} 1-\mathrm{S} 2=.06, p$ $<.001$; diferencia de medias S1-S3 $=.07, p<.001]$. Para esta variable no se encontraron interacciones significativas entre factores. El análisis de los TR tan solo mostró una interacción significativa entre los factores sexo $\mathrm{x}$ nivel HE para la sesión $2\left[F(1,35)=7.08, M S E=11432, p=.01, \eta^{2}=.17\right]$ y para el total de todas las sesiones $[F(1,35)=4.53, M S E=4249, p=.040$, $\left.\eta^{2}=.12\right]$, que se explicó por unos TR más largos en las niñas del grupo de $\uparrow \mathrm{HE}$ [TR-S2 ( $\uparrow \mathrm{HE}$ ): niños: $M=4.2$ seg.; niñas: $M=6.0$ seg.; diferencia de medias $=1.8$ seg., $p<.01$ ], [TR-TOTAL ( $\uparrow \mathrm{HE})$ : niños: $M=4.4$ seg.; niñas: $M$ $=5.6$ seg.; diferencia de medias $=1.2$ seg., $p=.01]$. También se observó un efecto marginal del factor sexo en la sesión $3[F(1,35)=4.05, M S E=8997$, 
$\left.p=.052, \eta^{2}{ }_{p}=.10\right]$, debido a los TR más cortos en niños que en niñas (niños: $M=4.7$ seg.; niñas: $M=5.6$ seg.; diferencia de medias = .9 seg., $p=.052$ ). No se encontraron otros efectos principales ni interacciones entre factores. El ANOVA de medidas repetidas 3 (sesión) 2 (sexo) x 2 (nivel de HE), tampoco mostró ningún efecto principal, ni interacciones entre factores. La Figura 2 muestra el rendimiento a través de las diferentes sesiones de entrenamiento en los estudiantes de Primaria.

\section{Tabla 1}

Estadísticos descriptivos (medias y desviaciones típicas) de la proporción de aciertos y de los TR de ensayos acertados (en segundos) en cada sesión y para el total de las tres sesiones del PERM en estudiantes de Primaria

Total $(\mathbf{N}=39)$

\begin{tabular}{ccccc}
\hline \multirow{2}{*}{ Medidas } & \multicolumn{2}{c}{$\downarrow$ HE $(\mathbf{n}=\mathbf{2 0})$} & \multicolumn{2}{c}{$\uparrow$ HE (n = 19) } \\
\cline { 2 - 5 } & Niños (n = 9) & Niñas (n = 11) & Niños (n = 8) & Niñas (n = 11) \\
\cline { 2 - 5 } & $\mathbf{M ~ ( D T )}$ & $\mathbf{M ~ ( D T )}$ & $\mathbf{M}(\mathbf{D T})$ & M (DT) \\
\hline AC-S1 & $.78(.13)$ & $.79(.15)$ & $.86(.06)$ & $.88(.08)$ \\
\hline AC-S2 & $.71(.13)$ & $.77(.12)$ & $.76(.12)$ & $.82(.10)$ \\
\hline AC-S3 & $.69(.15)$ & $.74(.13)$ & $.79(.11)$ & $.81(.11)$ \\
\hline AC-TOTAL & $.73(.13)$ & $.77(.13)$ & $.80(.09)$ & $.84(.09)$ \\
\hline TR-S1 & $5.2(1.3)$ & $4.6(1.1)$ & $4.6(.6)$ & $5.1(.9)$ \\
\hline TR-S2 & $5.4(.8)$ & $5.0(1.3)$ & $4.2(.8)$ & $6.0(1.8)$ \\
\hline TR-S3 & $4.8(1.5)$ & $5.5(2.0)$ & $4.5(.7)$ & $5.8(1.2)$ \\
\hline TR-TOTAL & $5.2(.7)$ & $5.0(1.3)$ & $4.4(.6)$ & $5.6(1.0)$ \\
\hline
\end{tabular}

La Tabla 2 muestra los análisis descriptivos de las puntuaciones obtenidas en el EFAI-E en ambos sexos y según el nivel en la habilidad espacial. El ANOVA de medidas repetidas 2 (pre-post) 2 (sexo) x 2 (nivel HE) para la variable dependiente "EFAI-E", solo mostró un efecto principal en el factor pre-post $\left[F(1,35)=79.1, M S E=827, p<.001, \eta_{p}^{2}=.69\right]$, explicando así una ganancia general en la habilidad de RM. No se encontraron otras interacciones significativas entre factores. 


\section{Figura 2}

Proporción de aciertos (A) y tiempo de respuesta de ensayos acertados (B) para la interacción Sesiones 1 a 3 x sexo x nivel de habilidad espacial en los estudiantes de Primaria
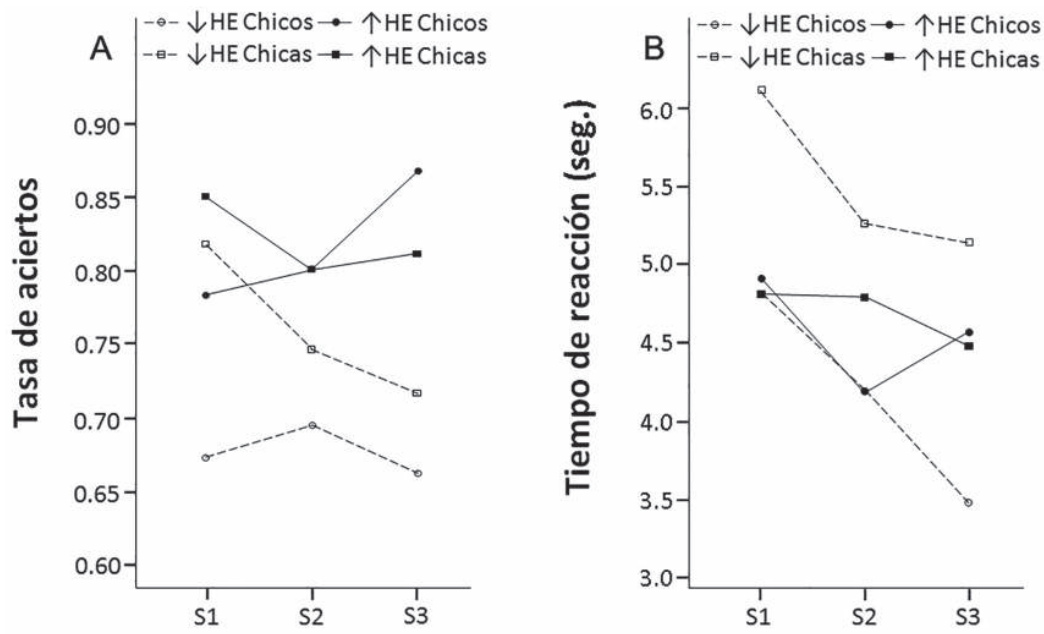

Tabla 2

Estadísticos descriptivos (medias y desviaciones típicas) de las puntuaciones obtenidas en el EFAI-E en ambos sexos del grupo de participantes de Primaria con bajo y alto nivel en la habilidad espacial

\begin{tabular}{|c|c|c|c|}
\hline \multicolumn{4}{|c|}{$\downarrow$ HE $(\mathrm{n}=20)$} \\
\hline \multirow{2}{*}{$\begin{array}{c}\text { EFAI-E } \\
(\text { máx. = 30) }\end{array}$} & $\operatorname{Niños}(n=9)$ & Niñas $(n=11)$ & TOTAL \\
\hline & M (DT) & M (DT) & M (DT) \\
\hline Pretest & $6.33(1.32)$ & $6.73(1.68)$ & $6.55(1.50)$ \\
\hline Postest & $14.4(5.13)$ & $13.3(4.59)$ & $13.8(4.74)$ \\
\hline Incremento & $8.11(4.70)$ & $6.55(4.80)$ & $7.25(4.70)$ \\
\hline \multicolumn{4}{|c|}{$\uparrow H E(n=19)$} \\
\hline \multirow{2}{*}{$\begin{array}{c}\text { EFAI-E } \\
(\text { máx. = 30) }\end{array}$} & Niños $(n=8)$ & Niñas $(n=11)$ & TOTAL \\
\hline & M (DT) & M (DT) & M (DT) \\
\hline Pretest & $13.6(3.82)$ & $12.0(3.13)$ & $12.7(3.43)$ \\
\hline Postest & $20.6(3.38)$ & $16.6(5.59)$ & $18.3(5.09)$ \\
\hline Incremento & $7.00(3.93)$ & $4.64(4.65)$ & $5.63(4.41)$ \\
\hline
\end{tabular}




\section{DISCUSIÓN}

El objetivo de este estudio fue examinar las diferencias individuales en el rendimiento a través de las diferentes sesiones de un programa de entrenamiento de RM, así como las ganancias diferenciales en función del nivel espacial de partida en escolares de $2^{\circ}$ curso de E.P.

Los resultados relativos a la proporción de aciertos mostraron, en general (cómputo global de las tres sesiones), que la proporción de aciertos fue mayor en el grupo de participantes con mayor capacidad espacial ( $\downarrow$ HE $=.75$ vs. $\uparrow \mathrm{HE}=.82$ ). En cualquier caso, no se obtuvo una interacción entre el momento de evaluación y el nivel de habilidad espacial, sugiriendo que ambos grupos tuvieron un rendimiento similar a través de las tres sesiones. Además, tanto niños como niñas tuvieron un rendimiento comparable en la proporción de aciertos a lo largo de todo el programa, por lo que no se encontró una interacción entre los factores sesión y sexo (niños $=.77$ vs. niñas $=.80$ ). Es interesante mencionar que la proporción de aciertos disminuyó progresiva y significativamente desde la primera a la tercera sesión, en ambos grupos según el nivel de habilidad espacial, y también en ambos sexos, posiblemente por el incremento progresivo en el nivel de dificultad de la tarea en cada una de las sesiones.

No se encontraron diferencias significativas en los TR de ambos grupos según la habilidad espacial inicial ( $\downarrow \mathrm{HE}=5.1 \mathrm{seg}$. vs. $\uparrow \mathrm{HE}=5.0 \mathrm{seg}$.), ni en función del sexo (niños $=4.8 \mathrm{seg}$. vs. niñas $=5.3 \mathrm{seg}$.). Este resultado podría estar en línea con los encontrados por Wiedenbauer y Jansen-Osmann (2008), que no encontraron en participantes de 10 y 11 años diferencias en TR entre niños y niñas. Sin embargo, los análisis mostraron que las niñas con mejores habilidades espaciales fueron las que necesitaron más tiempo para responder (TR significativamente mayores que el resto de los grupos).

En los estudios sobre diferencias de sexo en habilidades espaciales se han encontrado ciertas discrepancias. Algunos estudios sostienen que las intervenciones espaciales minimizan las diferencias de género (véase Linn y Hyde, 1989, para una revisión), mientras que otros no encuentran que la brecha de género en rendimiento espacial llegue a reducirse (Baenninger y Newcombe, 1989). Nuestros resultados convergen con los de estudios realizados en escolares donde se ha observado que ambos sexos se benefician en la misma medida tras un programa de entrenamiento en razonamiento espacial (Hawes et al., 2017; Lowrie et al., 2017). Los niños han mostrado incrementos similares a las niñas tras el entrenamiento espacial, y este hallazgo también se ha visto en ambos grupos según su nivel de habilidad espacial. 


\section{ESTUDIO 2}

\section{Método}

\section{Participantes}

Participaron 21 estudiantes $(M=14.3$ años, $D T=.44 ; 11$ chicos y 10 chicas) de tercer curso de Educación Secundaria del Colegio CEU-San Pablo de Montepríncipe (Boadilla del Monte, Madrid), con un nivel sociocultural medio-alto. Estos estudiantes pertenecían al GE de un estudio previo donde se comparó el rendimiento entre un grupo entrenado respecto a un grupo no entrenado (Rodán et al., 2016).

\section{Materiales}

Matrices progresivas de Raven (versión SPM; Raven et al., 1996)

Se trata del mismo instrumento utilizado y descrito en el Estudio 1, y su aplicación se llevó a cabo exactamente con el mismo procedimiento.

\section{Prueba de aptitud espacial "E" del EFAI-3}

Subprueba espacial de la batería Evaluación Factorial de Aptitudes Intelectuales (Santamaría et al., 2005) análoga a la descrita en el estudio de primaria. Para el estudio de las habilidades espaciales en este grupo se utilizó el Nivel 3, recomendado para participantes con edades entre 12 y 15 años. Este nivel consta de 27 ejercicios de evaluación más dos ejemplos de práctica (máximo 27 puntos). La tarea requiere que el participante combine la figura rotada de las opciones de respuesta con otras piezas ya colocadas en el target para que se complete el puzle. El tiempo de esta prueba es de seis minutos. Los estudios realizados por Santamaría et al. (2005) muestran índices de fiabilidad de .71.

\section{Programa de Entrenamiento de Rotación Mental (PERM)}

Esta versión del PERM consta de 330 ensayos de doble respuesta (lo que supone un total de 660 respuestas o decisiones). Cada presentación consiste en un molde blanco sobre una caja gris (target de referencia) situado en el lado izquierdo de la pantalla, y de dos figuras bidimensionales localizadas en el lado derecho - enumeradas como " 1 " y " 2 " — con una forma idéntica al molde (Figura 3). La dificultad dentro de cada sesión y entre sesiones se incrementó, manipulando la complejidad de las figuras, en cuanto a su simetría, número de estímulos, discontinuidad, etc. La tarea requiere que el participante imagine mentalmente rotaciones y señale si la figura "1" 
de la derecha encaja o no en el molde, y repetir la tarea con la figura " 2 ", de tal modo que, para acceder al ensayo consecutivo, el participante debía responder a cada figura registrándose finalmente un total de 600 respuestas de las 660 que se presentaban en el conjunto de las tres sesiones (los 10 primeros dobles ensayos de cada sesión eran de familiarización con la tarea). En cada sesión se registraron los aciertos y el tiempo de respuesta de cada ensayo (para información adicional sobre el PERM, véase el artículo de Rodán et al., 2016).

\section{Figura 3}

Ejemplo de un ensayo utilizado en el PERM del estudio 2
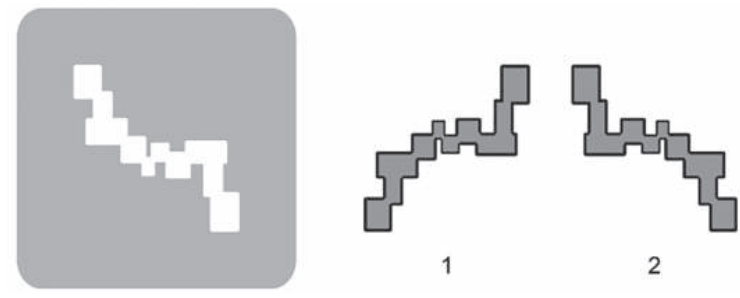

\section{Procedimiento}

Las pruebas se administraron en cinco días consecutivos, con una sesión por día dentro de una semana, distribuyéndose en tres fases bien diferenciadas.

Las pruebas Matrices Progresivas de Raven y EFAI-3 de la fase pretest se administraron colectivamente el primer día, en mesas individuales del aula habitual de los estudiantes, y supervisado por tres examinadores. El orden de las pruebas y el descanso entre estas fue el mismo que en el estudio 1.

El entrenamiento mediante el PERM se aplicó individualmente en la sala de informática durante tres sesiones consecutivas, una por día. Se realizaron 10 ensayos de prácticas para facilitar la comprensión de la tarea. El entrenamiento consistió en 100 presentaciones (200 decisiones) en cada sesión. Las instrucciones de los experimentadores a los participantes fueron las mismas que en el estudio 1 . El número de ensayos por sesión presentados en el PERM fue de 4200 (100 ensayos x 2 decisiones x 21 participantes). Tras la eliminación de algunos ensayos por anticipación o exceso de tiempo consumido, finalmente se analizaron un total de 3730 (88.8\%), 3778 (90.0 \%) y $3672(87.4 \%)$ en las sesiones S1, S2 y S3, respectivamente. Descontando estos ensayos eliminados, el tiempo mínimo de realización fue de $162 \mathrm{seg}$. (2.70 min.), $174 \mathrm{seg} .(2.90 \mathrm{~min}$.) y $180 \mathrm{seg}$. (3.00 min.), mientras que el tiempo máximo fue de $1146 \mathrm{seg}$. (19.1 min.), $684 \mathrm{seg}$. (11.4 min.) y $660 \mathrm{seg}$. (11.0 min.) para la S1, S2 y S3, respectivamente. 
Finalmente, en el último día, se administraron las pruebas Matrices Progresivas de Raven y EFAI-3 en las mismas condiciones que en la fase pretest.

Todos los tutores legales de los participantes firmaron un consentimiento informado y el estudio fue aprobado por el Comité de Ética de la UNED.

Análisis de datos

Los análisis realizados en este estudio fueron idénticos a los realizados en el Estudio 1.

\section{Resultados}

\section{Análisis preliminares}

Ambos grupos ( $\downarrow$ HE y $\uparrow \mathrm{HE}$ ) no difirieron en inteligencia en la fase previa. Tampoco se encontraron diferencias de sexo para todo el grupo, ni la interacción entre los factores grupo y sexo.

Rendimiento durante el PERM y ganancias obtenidas en la habilidad de RM

El nivel de HE ( $\downarrow$ HE y $\uparrow \mathrm{HE}$ ) de los chicos y chicas del GE se determinó de acuerdo a la mediana del EFAI-E en la fase pretest, que en este caso también fue de 9 puntos. Utilizando el mismo criterio que en el Estudio 1 , se excluyeron finalmente un total de 1420 ensayos (11.3\% del total). El promedio de TR en segundos se calculó en cada participante solo para las respuestas correctas.

La Tabla 3 muestra los análisis descriptivos de la proporción de aciertos y de los TR de los ensayos acertados. La proporción de aciertos en cada una de las tres sesiones (S1, S2, S3) y para el total de sesiones (TOTAL), el ANOVA mixto 2 (sexo) x 2 (nivel HE) reveló un efecto principal del sexo en la sesión $1\left[F(1,17)=5.02, M S E=.058, p=.039, \eta^{2}=.23\right]$, debido a una mayor proporción de aciertos en las chicas (chicas $M=.84$, chicos: $M=.73$, diferencia de medias $=.11, p=.039)$. También, se vio un efecto del nivel HE en la sesión $3\left[F(1,17)=12.4, M S E=.12, p=.003, \eta^{2}=.42\right]$, y para el total de todas las sesiones $\left[F(1,17)=6.64, M S E=.052, p=.020, \eta^{2}=.28\right]$, de forma que en ambos casos el grupo de participantes con alta HE obtuvo una mayor proporción de aciertos [AC-S3: $\downarrow$ HE: $M=.69$, $\uparrow$ HE: $M=.84$, diferencia de medias $=.15, p<.01$; AC-TOTAL: $\downarrow$ HE: $M=.72$, $\uparrow \mathrm{HE}: M=.82$, diferencia de medias $=.10, p=.02]$. El grupo de alta HE tuvo una mayor proporción 
de aciertos en la sesión 2, pero solo se encontró un efecto marginal [ $F$ (1, 17) $\left.=3.83, M S E=.032, p=.067, \eta^{2}=.18\right]$. No se encontraron otros efectos principales, ni interacciones. El ANOVA de medidas repetidas 3 (sesión) x 2 (sexo) x 2 (nivel HE) mostró una interacción significativa de los factores sesión x sexo $\left[F(2,34)=8.22, M S E=.016, p=.001, \eta^{2}{ }_{p}=.33\right]$, que se explicó por una disminución de la proporción de aciertos de la $\mathrm{S} 1$ a la $\mathrm{S} 3$ en el grupo de las chicas $[\mathrm{S} 1: M=.84 ; \mathrm{S} 2: M=.77 ; \mathrm{S} 3: M=.77$; diferencia de medias $\mathrm{S} 1-\mathrm{S} 2=.06, p<.01$; diferencia de medias $\mathrm{S} 1-\mathrm{S} 3=.07, p=.01]$. También se obtuvo una interacción significativa de los factores sesión x nivel HE $[F$ $\left.(2,34)=4.92, M S E=.010, p=.013, \eta_{p}^{2}=.22\right]$, de forma que solo el grupo de participantes con baja HE disminuyó la proporción de aciertos de la primera a la última sesión [S1: $M=.75 ; \mathrm{S} 3: M=.69$; diferencia de medias $\mathrm{S} 1-\mathrm{S} 3=.05, p=.04]$. Para esta variable no se encontraron otros efectos ni interacciones significativas. Para los TR, se encontró un efecto marginal de la interacción sexo x nivel HE en la sesión $3[F(1,17)=4.11, M S E=4000, p$ $\left.=.059, \eta_{p}^{2}=.20\right]$, que se explicó por unos TR menores en los chicos del grupo de baja HE y en las chicas del grupo de alta HE. No se encontraron otros efectos, ni interacciones significativas. El ANOVA de medidas repetidas 3 (sesión) x 2 (sexo) x 2 (nivel HE) mostró un efecto principal significativo del factor sesión, debido a que los TR decayeron de la S1 a la S3 [F (2, $\left.34)=4.62, M S E=3176, p=.017, \eta_{p}^{2}=.21\right]$. Las comparaciones por pares mostraron TR significativamente más largos en la S1 respecto a la S3 [S1: $M=5.2$ seg.; S3: $M=4.4$ seg.; diferencia de medias $\mathrm{S} 1-\mathrm{S} 3=.8, p=.05$ ]. No se encontraron otras interacciones significativas. La Figura 4 muestra el rendimiento a través de las diferentes sesiones de entrenamiento en los estudiantes de educación secundaria.

\section{Tabla 3}

Estadísticos descriptivos (medias y desviaciones típicas) de la proporción de aciertos y de los TR de ensayos acertados (en segundos) en cada sesión y para el total de las tres sesiones del PERM en estudiantes de Secundaria

\begin{tabular}{|c|c|c|c|c|}
\hline \multicolumn{5}{|c|}{ Total $(\mathrm{N}=21)$} \\
\hline \multirow[t]{3}{*}{ Medidas } & \multicolumn{2}{|c|}{$\downarrow$ HE $(n=11)$} & \multicolumn{2}{|c|}{$\uparrow H E(n=10)$} \\
\hline & Chicos $(n=6)$ & Chicas $(n=5)$ & Chicos $(n=5)$ & Chicas $(n=5)$ \\
\hline & M (DT) & M (DT) & M (DT) & M (DT) \\
\hline AC-S1 & $.67(.11)$ & $.82(.05)$ & $.78(.17)$ & $.85(.06)$ \\
\hline AC-S2 & $.70(.07)$ & $.75(.10)$ & $.80(.13)$ & $.80(.06)$ \\
\hline AC-S3 & $.66(.13)$ & $.87(.07)$ & $.72(.08)$ & $.81(.08)$ \\
\hline AC-TOTAL & $.68(.10)$ & $.76(.07)$ & $.82(.11)$ & $.82(.06)$ \\
\hline TR-S1 & $4.8(1.1)$ & $6.1(1.2)$ & $4.9(1.2)$ & $4.8(1.0)$ \\
\hline TR-S2 & $4.2(1.3)$ & $5.3(1.1)$ & $4.2(1.4)$ & $4.8(1.4)$ \\
\hline
\end{tabular}




\section{Total $(\mathbf{N}=21)$}

\begin{tabular}{ccccc}
\hline TR-S3 & $3.5(.7)$ & $5.1(.9)$ & $4.6(1.1)$ & $4.5(1.3)$ \\
\hline TR-TOTAL & $4.3(.8)$ & $5.5(.9)$ & $4.5(.8)$ & $4.6(1.0)$ \\
\hline
\end{tabular}

\section{Figura 4}

Proporción de aciertos (A) y tiempo de respuesta de los aciertos (B) para la interacción Sesiones 1 a 3 x sexo $x$ nivel de habilidad espacial en estudiantes de Secundaria
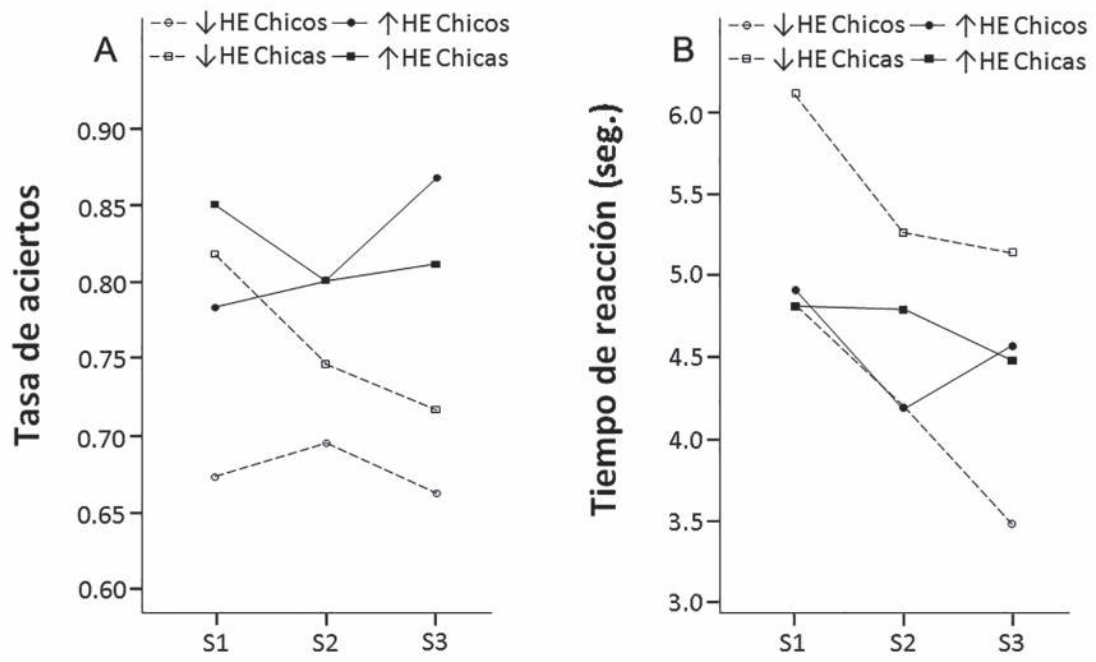

La Tabla 4 muestra los análisis descriptivos de las puntuaciones obtenidas en el EFAI-E en ambos sexos y según el nivel en la habilidad espacial. El ANOVA de medidas repetidas 2 (pre-post) x 2 (sexo) x 2 (nivel HE) para la variable dependiente "EFAI-E", mostró un efecto principal en el factor tiempo $\left[F(1,17)=88.1, M S E=32, p<.001, \eta^{2}{ }_{p}=.84\right]$, lo cual denotó una mejora, en general, de la habilidad de RM. No se encontraron otras interacciones significativas entre factores. 


\section{Tabla 4}

Estadísticos descriptivos (medias y desviaciones tipicas) de las puntuaciones obtenidas en el EFAI-E en ambos sexos del grupo de participantes de Secundaria con bajo y alto nivel en la habilidad espacial

\begin{tabular}{|c|c|c|c|}
\hline \multicolumn{4}{|c|}{$\downarrow$ HE (n = 11) } \\
\hline \multirow{2}{*}{$\begin{array}{c}\text { EFAI-E } \\
(\text { máx. = 30) }\end{array}$} & Chicos $(n=6)$ & Chicas $(n=5)$ & TOTAL \\
\hline & M (DT) & M (DT) & M (DT) \\
\hline Pretest & $7.50(1.05)$ & $7.40(.89)$ & $7.45(.93)$ \\
\hline Postest & $11.5(2.35)$ & $12.4(2.30)$ & $11.9(2.26)$ \\
\hline Incremento & $4.00(1.67)$ & $5.00(2.35)$ & $4.45(1.97)$ \\
\hline \multicolumn{4}{|c|}{$\uparrow \mathbf{H E}(\mathrm{n}=10)$} \\
\hline \multirow{2}{*}{$\begin{array}{c}\text { EFAI-E } \\
(\text { máx. = 30) }\end{array}$} & Chicos $(n=5)$ & Chicas $(n=5)$ & TOTAL \\
\hline & M (DT) & M (DT) & M (DT) \\
\hline Pretest & $12.8(3.19)$ & $12.2(1.48)$ & $12.5(2.37)$ \\
\hline Postest & $20.2(3.03)$ & $18.0(1.87)$ & $19.1(2.64)$ \\
\hline Incremento & $7.40(3.58)$ & $5.80(3.03)$ & $6.60(3.24)$ \\
\hline
\end{tabular}

\section{DISCUSIÓN}

En general se encontró en el grupo de estudiantes con mejor habilidad en la prueba espacial "E" del EFAI-3 una proporción de aciertos significativamente mayor en el PERM respecto al grupo con baja habilidad espacial. El análisis individualizado de cada sesión solo mostró diferencias significativas entre estos grupos en la última sesión. Además, se vio una interacción tiempo por nivel de HE en la proporción de aciertos, de forma que los participantes con mejores HE mejoraron su rendimiento desde la primera hasta la tercera sesión, mientras que aquellos con bajas HE le disminuyeron a lo largo de las tres sesiones.

También se observó una mayor proporción de aciertos en el grupo de chicas para la muestra total del grupo experimental. Sin embargo, las chicas solo difirieron significativamente de los chicos en la primera sesión de entrenamiento. Además, se encontró una interacción de sesión por sexo, debido a que el grupo de chicas experimentó una disminución significativa en la proporción de aciertos desde la primera sesión a la última. Otro hallazgo fue que los chicos con mejores HE incrementaron su proporción de aciertos conforme avanzaba el programa, aunque no se vieron diferencias significativas de sexo entre grupos de alta y baja capacidad espacial a través de las diferentes sesiones de entrenamiento. 
Aunque solo los estudiantes con peor capacidad espacial en el EFAI-3 experimentaron una disminución significativa de los TR desde la primera a la última sesión del PERM, ambos grupos de alta y baja habilidad espacial, tuvieron un rendimiento similar a lo largo del entrenamiento en esta variable. No se encontraron diferencias en los TR de niños y niñas, en línea con los hallazgos de Wiedenbauer y Jansen-Osmann (2008) que tampoco vieron en escolares un efecto de género en los tiempos de respuesta en una tarea de RM.

Nuestros resultados en población adolescente convergen con los hallados en otras investigaciones llevadas a cabo en el mismo grupo de edad, que sostienen que ambos sexos se benefician de manera similar tras un programa de entrenamiento en razonamiento espacial (Neubauer et al., 2010; Samsudin et al., 2011; Sanz de Acedo Lizarraga y García Ganuza, 2003). Respecto a los incrementos producidos con el entrenamiento, nuestro estudio es convergente con otras investigaciones que no encontraron ganancias diferenciales según el nivel de partida en intervenciones con preescolares de 3 años (Fernández-Méndez et al., 2020) o con adultos universitarios (Sims y Mayer, 2002).

\section{DISCUSIÓN GENERAL Y CONCLUSIONES}

Cualquier intervención de las habilidades espaciales que ocurra antes o durante la adolescencia podría ser crucial, fundamentalmente cuando la presencia de personas con menos recursos espaciales (incluido el sexo femenino) es escasa en ciertos grados universitarios relacionados con las ciencias, la tecnología, la ingeniería y las matemáticas. Por ello, y de acuerdo a lo sugerido por Uttal et al. (2013) en su influyente meta-análisis sobre maleabilidad de habilidades espaciales, realizamos una investigación utilizando un método análogo en dos grupos de edad distintos — primaria y secundaria-, tanto para la evaluación pre-post, como para el entrenamiento en RM, para así poder hacer comparables los resultados obtenidos a lo largo de tres sesiones de entrenamiento. El objetivo de estas dos investigaciones fue analizar la ejecución de la tarea a lo largo de un entrenamiento en RM mediante el PERM, examinando el rendimiento de los participantes por sexos y por niveles de habilidad espacial (baja y alta HE en la prueba de aptitud espacial "E" del EFAI-3). También se valoraron las ganancias diferenciales producidas después del entrenamiento según el género y el nivel espacial de partida. Basándonos en la literatura previa, esperábamos que los participantes con menores habilidades espaciales iniciales experimentasen mayores ganancias hasta alcanzar un nivel similar tras el entrenamiento a aquellos participantes con mayores recursos espaciales. 
En general (cómputo global de las tres sesiones), solo hubo diferencias en la proporción de aciertos, de manera que esta fue menor en participantes con bajas HE. No obstante, los estudiantes con "baja" HE tuvieron buena ejecución, alcanzando una proporción de aciertos a través de las tres sesiones de entrenamiento del 0.75 y del 0.72 , en los estudiantes de primaria y secundaria, respectivamente. En cualquier caso, estos resultados difieren significativamente de los obtenidos por los participantes con altas habilidades espaciales ( 0.82 en los estudiantes entrenados de primaria y secundaria).

Los análisis de evolución a lo largo de las tres sesiones también mostraron ciertos puntos de convergencia y de divergencia entre los dos grupos de edad. En el grupo de escolares, la proporción de aciertos decayó significativamente y sus respuestas fueron más lentas desde la primera a la última sesión, aunque no podemos concluir si fue debido al incremento de la dificultad de la tarea conforme avanzaba el programa de entrenamiento o a una falta de motivación. En cualquier caso, en general, el nivel de aciertos en escolares fue bueno (0.83 en la sesión 1; 0.76 en la sesión 3). En el grupo de adolescentes, sin embargo, disminuyeron significativamente los TR desde la primera sesión (5.2 seg.) hasta la última (4.4 seg.), manteniendo prácticamente constante su proporción de aciertos a lo largo de las tres sesiones $(\approx 0.77)$, lo que podría denotar que, a pesar del incremento en la dificultad de la tarea con las sesiones, su nivel de motivación y/o su aprendizaje les permitía responder más rápido y mantener un nivel óptimo de aciertos.

Los perfiles de proporción de aciertos sí mostraron diferencias en ambos grupos de edad, encontrándose únicamente variaciones a través de las tres sesiones por grupos de sexo y de HE en los adolescentes. Por grupos de sexo, los chicos mejoraron su habilidad de RM desde la primera sesión a la última, mientras que las chicas empeoraron desde el comienzo al final del programa de entrenamiento. Estos resultados podrían ser explicados porque los chicos estuvieran más motivados por producir respuestas correctas a lo largo de las diferentes sesiones del PERM, similar a los resultados encontrados por Voyer et al. (2004), que sugirieron que los hombres parecen estar más motivados por la necesidad de producir respuestas, mostrando un menor comportamiento de adivinación de respuestas.

Un aspecto escasamente valorado en la literatura sobre entrenamiento en RM usando un método análogo en diferentes edades, es el de los incrementos producidos en función del nivel espacial inicial. En este trabajo se han encontrado resultados similares en ambos grupos de edad, de forma que los participantes con baja habilidad espacial se beneficiaron en una proporción similar a aquellos participantes con mejores capacidades 
espaciales. Estos hallazgos son compatibles con los resultados de estudios sobre diferencias de género en habilidades espaciales, en los que se ha observado que ambos sexos se benefician en la misma medida tras un entrenamiento en razonamiento espacial, tanto en escolares de primaria (Hawes et al., 2017; Lowrie et al., 2017) como en adolescentes de secundaria (Neubauer et al., 2010; Samsudin et al., 2011; Sanz de Acedo Lizarraga y García Ganuza, 2003). Nuestros resultados también convergen con los obtenidos en algunas investigaciones que no han encontrado ganancias diferenciales tras un entrenamiento espacial según el nivel de partida en RM en preescolares de 3 años (Fernández-Méndez et al., 2020) y adultos universitarios (Sims y Mayer, 2002).

Finalmente, merece la pena mencionar la variabilidad en el tiempo invertido de la tarea de entrenamiento en ambos estudios, lo cual pone de manifiesto que algunos participantes necesitan un tiempo sustancialmente superior para alcanzar un rendimiento óptimo. Esto es importante de cara al diseño de programas adaptativos de entrenamiento, de manera que, para acceder a un nivel de dificultad superior, debería alcanzarse un mínimo que asegure que los ítems más básicos han sido asimilados. Solamente así este tipo de entrenamientos podrían ganar en eficacia y tener una finalidad educativa, especialmente en aquellos participantes que tienen menos recursos.

\section{Limitaciones del estudio y futuras líneas de investigación}

El pequeño número de participantes en ambos estudios, especialmente en secundaria $(\mathrm{N}=21)$, podría haber afectado a los análisis de varianza, por lo que debemos ser cautelosos en la generalización de las conclusiones obtenidas. En este sentido, sería deseable que futuras investigaciones contaran con una muestra más numerosa para asegurar la generalización de resultados.

El programa de entrenamiento en nuestra opinión no carece de intensidad (450 y 600 ensayos en escolares y adolescentes, respectivamente), y en general los participantes mostraron un alto nivel de motivación y competitividad con sus pares para rendir al máximo. Sin embargo, tres sesiones podrían haber sido insuficientes para mostrar mayores incrementos en los individuos con baja habilidad espacial. En este sentido, Terlecki et al. (2008) hicieron un seguimiento de doce semanas de entrenamiento espacial, encontrando que los participantes con menores habilidades espaciales necesitaban algún tiempo para mostrar ganancias espaciales y equipararse a los participantes con mejores capacidades. En futuros estudios, el análisis de la relación entre el tiempo total que lleva realizar la tarea (incluyendo 
ensayos que no se aciertan) y la proporción de aciertos, así como el análisis de la progresión a lo largo de los ensayos permitiría conocer el perfil de evolución en el aprendizaje de un estudiante, en función del tiempo total, cuando se realiza este tipo de entrenamiento.

A pesar de estas limitaciones, pensamos que el estudio resulta de interés para establecer directrices en diseño de programas de mejora de la cognición espacial, promoviendo e implementando entrenamientos adaptativos y personalizados para ayudar a los estudiantes con más déficit.

En los dos estudios de este trabajo se ha utilizado una tarea de entrenamiento en RM encuadrada en la categoría intrínseca-dinámica (véase Uttal et al., 2013) y que supone una manipulación o transformación en un marco de referencia egocéntrico. Sin embargo, el campo de las habilidades espaciales podría implicar el uso de contextos bien diferentes, como los extrínsecos-dinámicos, e incluso de tipo alocéntrico, donde el marco de referencia respecto al estímulo que se rota es externo. Este tipo de escenarios espaciales es utilizado con frecuencia por pilotos de drones o controladores aéreos. En este sentido, sería interesante valorar hasta qué punto puede influir un entrenamiento con este tipo de tareas de transformación mental en la adquisición de estrategias para llevar a cabo óptimamente problemas relacionados con dominios STEM, donde para llegar a una solución podría necesitarse el uso de este tipo de representaciones mentales.

\section{NOTAS}

Financiación: El presente trabajo ha sido financiado en parte con el proyecto RTI2018-098523-B-I00 “Aprendizaje de habilidades visoespaciales para favorecer la igualdad de oportunidades desde las perspectivas educativas, evolutivas y de género" del Ministerio de Ciencia, Innovación y Universidades.

Agradecimientos: Los autores agradecen a los C.E.I.P "Escuelas Aguirre" y "Leopoldo Alas" de Madrid y al Colegio CEU-San Pablo Montepríncipe, por poner a disposición la muestra de participantes y las instalaciones para realizar las tareas. También, a Patricia Gimeno (C.E.I.P "Leopoldo Alas"), por abrirnos las puertas de manera incondicional y su ayuda con la recopilación de datos. 


\section{REFERENCIAS BIBLIOGRÁFICAS}

Baenninger, M., \& Newcombe, N. (1989). The role of experience in spatial test performance: A meta-analysis. Sex Roles, 20(5-6), 327-344. https://doi. org/10.1007/BF00287729

Baniqued, P. L., Kranz, M. B., Voss, M. W., Lee, H., Cosman, J. D., Severson, J., \& Kramer, A. F. (2014). Cognitive training with casual video games: points to consider. Frontiers in Psychology, 4, 1010. https://doi. org/10.3389/fpsyg.2013.01010

Bergner, S., \& Neubauer, A. C. (2011). Sex and training differences in mental rotation: A behavioral and neurophysiological comparison of gifted achievers, gifted underachievers and average intelligent achievers. High Ability Studies, 22(2), 155-177. https:// doi.org/10.1080/13598139.2011.6288 49

Cherney, I. D., Bersted, K., \& Smetter, J. (2014). Training spatial skills in men and women. Perceptual \& Motor Skills, 119(1), 82-99. https://doi. org/10.2466/23.25.PMS.119c12z0

Contreras, M. J., Escrig, R., Prieto, G., \& Elosúa, M. R. (2018). Spatial Visualization ability improves with and without studying Technical Drawing. Cognitive Processing, 19(3), 387-397. https://doi.org/10.1007/ s10339-018-0859-4

David, L. T. (2012). Training effects on mental rotation, spatial orientation and spatial visualisation depending on the initial level of spatial abilities. Procedia-Social and Behavioral Sciences, 33, 328-332. https://doi. org/10.1016/j.sbspro.2012.01.137

De Lisi, R., \& Wolford, J. (2002). Improving children's mental rotation accuracy with computer game playing. Journal of Genetic Psychology, 163(3), 272-283. https:// doi.org/10.1080/00221320209598683

Ehrlich, S. B., Levine, S. C., \& GoldinMeadow, S. (2006). The importance of gesture in children's spatial reasoning. Developmental Psychology, 42(6), 12591268. https://doi.org/10.1037/00121649.42.6.1259

Fernández-Méndez, L. M., Contreras, M. J., \& Elosúa, M. R. (2020). Developmental differences between 1 st and 3rd year of early childhood education (preschool) in mental rotation and its training. Psychological Research, 84(4), 1056-1064. https://doi. org/10.1007/s00426-018-1104-6

García-Madruga, J. A., Elosúa, M. R., Gil, L., Gómez-Veiga, I., Vila, J. Ó., Orjales, I.,.., \& Duque, G. (2013). Reading comprehension and working memory's executive processes: An intervention study in primary school students. Reading Research Quarterly, 48(2), 155-174. https://doi.org/10.1002/ rrq. 44

Hawes, Z., Moss, J., Caswell, B., Naqvi, S., \& MacKinnon, S. (2017). Enhancing children's spatial and numerical skills through a dynamic spatial approach to early geometry instruction: effects of a 32-week intervention. Cognition and Instruction, 35(3) 1-29. https://doi.org /10.1080/07370008.2017.1323902

Humphreys, L. G., Lubinski, D., \& Yao, G. (1993). Utility of predicting group membership and the role of spatial visualization in becoming an engineer, physical scientist, or artist. Journal of Applied Psychology, 78(2), 250-261. https://doi.org/10.1037/00219010.78.2.250. 
Karbach, J., Strobach, T., \& Schubert, T. (2015). Adaptive working-memory training benefits reading, but not mathematics in middle childhood. Child Neuropsychology, 21(3), 285301. https://doi.org/10.1080/09297049 .2014 .899336

Linn, M. C., \& Hyde, J. S. (1989). Gender, mathematics, and science. Educational Researcher, 18(8), 17-27. https://doi. org/10.3102/0013189X018008017

Linn, M. C., \& Petersen, A. C. (1985). Emergence and characterization of sex differences in spatial ability: A meta-analysis. Child Development, 56(6), 1479-1498. https://doi. org/10.1111/j.1467-8624.1985. tb00213.x

Lohman, D. F. (1996). Spatial ability and g. In I. Dennis \& P. Tapsfield (Eds.), Human abilities: Their nature and measurement (pp. 97-116). Lawrence Erlbaum Associates, Inc.

Lowrie, T., Logan, T., \& Ramful, A. (2017). Visuospatial training improves elementary students' mathematics performance. British Journal of Educational Psychology, 87(2), 170186. https://doi.org/10.1111/bjep. 12142

Neubauer, A. C., Bergner, S., \& Schatz, M. (2010). Two-vs. three-dimensional presentation of mental rotation tasks: Sex differences and effects of training on performance and brain activation. Intelligence, 38(5), 529-539. https:// doi.org/10.1016/j.intell.2010.06.001

Rafi, A., Samsudin, K. A., \& Said, C. S. (2008). Training in spatial visualization: The effects of training method and gender. Educational Technology \& Society, 11(3), 127-140.

Raven, J., Court, J. H. \& Raven, J. C. (1996). Standard Progressive Matrices. Psychologists Press.
Rodán, A., Contreras, M. J., Elosúa, M. R., \& Gimeno, P. (2016). Experimental but not sex differences of a mental rotation training program on adolescents. Frontiers in Psychology, 7, 1050. https://doi.org/10.3389/ fpsyg.2016.01050

Rodán, A., Gimeno, P., Elosúa, M. R., Montoro, P. R., \& Contreras, M. J. (2019). Boys and girls gain in spatial, but not in mathematical ability after mental rotation training in primary education. Learning and Individual Differences, 70, 1-11. https://doi. org/10.1016/j.lindif.2019.01.001

Samsudin, K., Rafi, A., \& Hanif, A. S. (2011). Training in mental rotation and spatial visualization and its impact on orthographic drawing performance. Journal of Educational Technology \& Society, 14(1), 179-186.

Santamaría, P., Arribas, D., Pereña, J., \& Seisdedos, N. (2005). EFAI, Evaluación Factorial de las Aptitudes Intelectuales. Departamento I+D TEA Ediciones.

Sanz de Acedo Lizarraga, M. L., \& García Ganuza, J. M. (2003). Improvement of mental rotation in girls and boys. Sex Roles, 49(5-6), 277-286. https://doi. org/10.1023/A:1024656408294

Seisdedos, N. (1995). Manual de las Matrices Progresivas de Raven. Escalas $C P M$, SPM y APM. TEA Ediciones.

Shepard, R. N., \& Metzler, J. (1971). Mental rotation of three-dimensional objects. Science, 171(3972), 701703. https://doi.org/10.1126/ science.171.3972.701

Sims, V. K., \& Mayer, R. E. (2002). Domain specificity of spatial expertise: The case of video game players. Applied Cognitive Psychology: The Official Journal of the Society for Applied Research in Memory and Cognition, 
16(1), 97-115. https://doi.org/10.1002/ acp.759

Terlecki, M. S., Newcombe, N. S., \& Little, M. (2008). Durable and generalized effects of spatial experience on mental rotation: Gender differences in growth patterns. Applied Cognitive Psychology: The Official Journal of the Society for Applied Research in Memory and Cognition, 22(7), 996-1013. https://doi. org/10.1002/acp.1420

Uttal, D. H., Meadow, N. G., Tipton, E., Hand, L. L., Alden, A. R., Warren, C., \& Newcombe, N. S. (2013). The malleability of spatial skills: A meta-analysis of training studies. Psychological Bulletin, 139(2), 352402. https://doi.org/10.1037/a0028446

Voyer, D., Rodgers, M. A., \& McCormick, P. A. (2004). Timing conditions and the magnitude of gender differences on the Mental Rotations Test. Memory \& Cognition, 32(1), 72-82. https://doi. org/10.3758/BF03195821

Voyer, D. \& Jansen, P. (2016). Sex differences in chronometric mental rotation with human bodies. Psychological Research, 80(6), 974984. https://doi.org/10.1007/s00426015-0701-x

Wiedenbauer, G., \& Jansen-Osmann, P. (2007). Mental rotation ability of children with spina bifida: what influence does manual rotation training have? Developmental Neuropsychology, 32(3), 809-824. https://doi. org/10.1080/87565640701539626

Wiedenbauer, G., \& Jansen-Osmann, P. (2008). Manual training of mental rotation in children. Learning and Instruction, 18(1), 30-41. https://doi. org/10.1016/j.learninstruc.2006.09.009 


\section{PERFIL ACADÉMICO Y PROFESIONAL DE LOS AUTORES}

Antonio Rodán González. ORCID: https://orcid.org/0000-0001-7674-3335

Profesor Adjunto del Área de Óptica y Optometría en el Dpto. de Química y Bioquímica. Facultad de Farmacia de la Universidad San Pablo CEU. Su interés investigador se centra en el estudio de a) la función visual en pacientes con anomalías visuales (refractivas y funcionales), b) procesos y estrategias viso-espaciales y c) plasticidad de las habilidades espaciales a través de programas de entrenamiento. E-mail: antonio.rodangonzalez@ceu.es

Pedro Raúl Montoro Martínez. ORCID: https://orcid.org/0000-0002-5665-8587

Profesor Titular de Universidad del Departamento de Psicología Básica I en la Facultad de Psicología de la UNED. Su área de especialización se ubica en la confluencia de la Psicología experimental y la Neurociencia Cognitiva, con especial atención a los mecanismos cognitivos y neurales de los procesos de organización perceptiva, reconocimiento de objetos, memoria visual, atención selectiva y consciencia visual. E-mail: prmontoro@psi.uned.es

Agustín Martínez Molina. ORCID: https://orcid.org/0000-0001-5251-6066

Profesor asociado del Departamento de Psicología Social y Metodología en la Facultad de Psicología de la Universidad Autónoma de Madrid. Especialización en (a) desarrollo de test de inteligencia y personalidad, y en (b) métodos estadísticos para el modelado de datos (p.ej., factoriales y ecuaciones estructurales). E-mail: agustin.martinez@uam.es

María José Contreras Alcalde. ORCID: https://orcid.org/0000-0001-6302-5238

Profesora Titular de Universidad del Departamento de Psicología Básica I en la Facultad de Psicología de la UNED. Su interés investigador se centra en la plasticidad de las capacidades visoespaciales, y su desarrollo mediante el entrenamiento. Especialización en el análisis del rendimiento visoespacial a lo largo de distintas etapas de desarrollo, así como el efecto que los programas de entrenamiento tienen para conseguir la equidad, reduciendo las diferencias entre sexos tradicionalmente encontradas en este tópico. E-mail: mjcontreras@psi.uned.es

Fecha Recepción del Artículo: 23. Febrero. 2021

Fecha Modificación del Artículo: 24. Junio. 2021

Fecha Aceptación del Artículo: 30. Junio. 2021

Fecha Revisión para Publicación: 13. Septiembre. 2021 